\title{
Automatic Categorization of Traditional Chinese Painting Images with Statistical Gabor Feature and Color Feature
}

\author{
Xiaohui Guan, Gang Pan, and Zhaohui Wu \\ Dept. of Computer Science, Zhejiang University, Hangzhou, China \\ $\{$ hxgy, gpan, wzh\}@zju.edu.cn
}

\begin{abstract}
This paper presents an automatic statistical approach to categorize traditional Chinese painting (TCP) images according to subject matter into three major classes: figure paintings, landscapes, and flowerand-bird paintings. A simple statistical Gabor feature is presented to describe the local spatial configuration of the image, which is then integrated with color histogram that represents the global visual characteristic to build the feature subspace. A relative-distance based voting rule is proposed for final classification decision. The effectiveness of the proposed scheme is demonstrated by the comparable experimental results.
\end{abstract}

\section{Introduction}

Traditional Chinese painting (TCP) is highly regarded throughout the world for its theory, expression, and techniques. As an important part of Chinese cultural heritage, the traditional Chinese painting is distinguished from Western art in that it is executed on xuan paper (or silk) with the Chinese brush, Chinese ink and mineral and vegetable pigments. Currently more and more museums and artists like to present the paintings on the Web. This paper addresses the automatic categorization of the traditional Chinese painting images. The potential applications include Web searching and browsing, digital art libraries etc.

Traditional Chinese painting can be divided according to subject matter into three major categories [15]: landscape paintings, flower-and-bird paintings and figure paintings. Landscape paintings mainly depict the natural scenery of mountains and rivers. Flower-and-bird paintings concentrate on the plants and small animals. Figure paintings mainly portray humans and their activities, generally along with the plant, small animal and scenery. Although the categorization is usually an easy task for humans, it is an extremely difficult problem for machines. The major difficulties lie in its wide variation in subject's shape, color, and painting style. The subjects' appearance in TCP is usually highly abstracted from the real word.

There are many researchers made efforts for image classification and indexing. As one of the simplest representations of digital images, color histogram [1] has been widely used for various image categorization problems. Color histogram 
is insensitive to image resolution and rigid transform. Pass [2] presents color coherence vector (CCV) as image index. CCV is the statistical pixels' convergence measurement in space. Stricker and Orengo think that the color information centralizes in the low rank color moments, so they use the statistical moment to characterize the color [3]. The above methods attempt to capture the global color features and discard the spatial configuration. The reference [4] utilizes spatial information to improve the histogram method.

Besides histogram, a number of block-based methods have been proposed to employ local and spatial properties by dividing an image into rectangular subimages. [5] exploits the wavelet coefficients in high frequency bands for classification. Ma and Manjunath evaluate various wavelet forms to reach a conclusion that Gabor wavelet transforms get the best results in texture retrieval methods [6]. Yu [7] uses 1D HMM to classify indoor and outdoor scene. Carson et al [8] utilizes EM algorithm to segment the image into several "blobworld" which are coherent in color and texture. Huang [9] relies on the banded color correlogram as image features and then hierarchical classification tree is construct to classify new image. Jiang [10] presents a scheme to classify the TCP images from non-TCP images.

This paper proposes a statistical method to automatically classify the traditional Chinese paintings according to subject matter into three major categories: landscape paintings, flower-and-bird paintings and figure paintings. We use a statistical Gabor feature as the representation of local texture of TCP, and color histogram as the global representation of color information.

\section{Statistical Feature Representation}

In our method, we present a statistical feature integrating local texture and color information. Firstly, the TCP image is filtered using a bank of Gabor functions with multiple scales $v$ and orientations $u$. Secondly, with the filtered image, we construct a statistical Gabor feature such as average, variance. And then, we combine the Gabor feature with the color histogram of the TCP.

\subsection{Statistical Gabor Feature}

Gabor wavelets are biologically motivated because they model the response properties of human cortical cells. They have been widely adopted to extract features in image retrieval, face recognition, texture classification $[11,12]$ and got wonderful results. Basically, Gabor filters are a group of wavelets, with each wavelet capturing the variation at a specific frequency and direction. It has been shown to correspond to human visual system.

Gabor filter family is similar to the simple visual perceptive cells, which are characterized as localized orientation selective and frequency selective. The kernel is the product of a Gaussian envelope and a wave function, as shown in Equ (1), where the term $\exp \left(-\frac{\delta^{2}}{2}\right)$ is subtracted to make the filter insensitive to the overall level of illumination. 


$$
\Phi_{j}(\vec{x})=\frac{\left\|\overrightarrow{k_{j}}\right\|}{\delta^{2}} \exp \left(-\frac{\left\|\overrightarrow{k_{j}}\right\| \bullet\|\vec{x}\|}{\vec{x}}\right)\left[\exp \left(i \overrightarrow{k_{j}} \vec{x}\right)-\exp \left(-\frac{\delta^{2}}{2}\right)\right]
$$

Where

$$
\begin{gathered}
\vec{x}=I(x, y) \quad \overrightarrow{k_{j}}=\left[\begin{array}{l}
k_{\nu} \cos \varphi_{\mu} \\
k_{\nu} \sin \varphi_{\mu}
\end{array}\right] \\
k_{v}=\frac{\Pi}{2} * \frac{1}{2^{\nu}} \quad \varphi_{\mu}=\mu \frac{\Pi}{6} \quad \nu=0 \cdots 3, \mu=0 \cdots 5
\end{gathered}
$$

On basis of [13], the parameters setting of Gabor filter are 4 scales, 6 orientations, and filter mask size is $13 \times 13$. So we get 24 functions determined by the parameters $\nu, \mu$. This bank of filters is applied to the TCP image, shown in Equ (2). This means Gabor wavelet transformation, that is, every input image is convoluted with the 24 Gabor filters.

$$
G_{\nu \mu}(\vec{x})=\int I(\vec{y}) \Phi_{\nu \mu}(\vec{x}-\vec{y}) d^{2} \vec{y}
$$

The resulted Gabor coefficient has two parts: real part $\operatorname{Re}\left(G_{\nu \mu}\right)$ and imaginary part $\operatorname{Im}\left(G_{\nu \mu}\right)$. From the filtered image, we could compute the average $\varrho_{\nu \mu}$ and variance $\sigma_{\nu \mu}$ of the magnitude $\sqrt{\operatorname{Re}\left(G_{\nu \mu}\right)^{2}+\operatorname{Im}\left(G_{\nu \mu}\right)^{2}}$, then obtain the statistical Gabor feature, a 48-dimensional vector:

$$
\vec{g}=\left\{\varrho_{00}, \sigma_{00}, \varrho_{01}, \sigma_{01} \ldots \varrho_{\nu \mu} \sigma_{\nu \mu} \mid \nu=3, \mu=5\right\}
$$

\subsection{Statistical Color Feature}

Color is the most intuitive characteristic of human vision. Every kind of subjects has its different intrinsic color. We employ the color histogram [1] as our statistical feature for color information, which has been used in the image retrieval and indexing. It is invariant to translation and rotation around the viewing axis and varies slowly with changes of view angle, scale and occlusion.

Assume colors in an image are mapped into a discrete color space containing $n$ colors, a color histogram of image $I$ is n-dimensional vector, where each element represents the number of pixels with color index $j$. Each element of a histogram is normalized so that the histogram represents the image without regarding to the image size. The element of the normalized color histogram $h(I)$ is defined as:

$$
h_{j}(I)=H_{j}(I) / \sum_{i=1}^{n} H_{i}(I)
$$

Where $H_{i}(I), H_{j}(I)$ is the number of pixels with color $i, j$ in image $I$. In our experiment, we select $8 \times 8 \times 8 \mathrm{RGB}$ color space. So we get a color feature vector:

$$
\vec{h}=\left\{h_{j}(I) \mid j=1 \cdots 512\right\}
$$

We combine the color histogram $\vec{h}$ with the Gabor feature $\vec{g}$ into a longer vector $\{\vec{g} \mid \vec{h}\}$, which together characterizes the texture and color of TCP image. 


\section{Categorization}

\subsection{Voting with Relative-Distance in Feature Space}

To classify a new input image $I$ in a feature space, we exploit voting with sample images. In many articles, Euclidean distance is used. However, for the traditional Chinese painting images, works in different style may have different dispersibility. The Euclidean distance in the feature space is often not enough, since the scatter information of the same class should be taken into account.

Here we take the intra-class variance into consideration. Suppose that $X=$ $\left\{x_{1}, x_{2} \cdots x_{N}\right\}, \quad Y_{m k}=\left\{y_{1}, y_{2} \cdots y_{N}\right\}$, where $X$ is the feature vector of the input image $I, Y_{m k}$ is $k$ th sample feature vector of class m. $K_{m}$ is the total number of training images of class $\mathrm{m}$. We use the Equ (6) to determine the distance between the input image and the class $m$.

$$
\bar{d}_{m}=\sum_{i=1}^{K_{m}} d_{m k} / K_{m} \quad v_{m}=\sqrt{\sum_{k=1}^{K_{m}}\left(d_{m k}-\bar{d}_{m}\right)^{2} / K_{m}}
$$

And finally the relative-distance between the input image $I$ and the $k$ th sample is:

$$
R d_{m k}=d_{m k} / v_{m} \quad d_{m k}=d\left(X, Y_{m k}\right)=\sqrt{\sum_{n=1}^{N}\left(x_{i}-y_{i}\right)^{2}}
$$

We make the relative distance to decide whether to cast a vote of a certain class to the input image. In most cases the relative distance performs better than the common Euclidean measure.

The voting policy is according to the following rule:

$$
V_{m}=\sum_{k=1}^{K_{m}} V_{m k} \quad V_{m k}= \begin{cases}1 & \text { if } R d_{m k}<\text { Threshold } \\ 0 & \text { otherwise }\end{cases}
$$

$V_{m}$ is the total number of votes received by the class m. Assuming $C_{m}$ is the total samples of the class m. Thus, maximum $V_{m} / C_{m}$ determines the pattern of test image.

\subsection{Sample Selection}

In order to refine the training samples and also to improve the classification accuracy, sample selection procedure is implemented. The selection of training samples may affect completely the categorization results, because images to classify need be compared with all the training samples in the reference database. Fig.1.(a) shows two classes of traditional Chinese painting image, where blue star denotes the flower-and-bird paintings and red point denotes figure paintings. Each painting is represented by a point in the feature space.

We use the $k$-nearest neighbor editing algorithm [14] to select the registered samples from a set of reference samples. The algorithm is as follows: 
1. Divide randomly reference sample set into several subsets, suppose: $\Theta=$ $\left\{S_{0}, S_{1} \cdots S_{n-1}\right\}, \quad n \geq 3$,

2. With $S_{(i+1) \bmod (n)}$ as reference samples, Use $K$-nearest classification method to classify $S_{i}$ samples, which $i=0,1 \cdots n-1$.

3. Discard classified falsely samples, the residual samples constitute new sample set $\Theta^{N}$.

4. If there are no samples to be discarded, stop. Otherwise, go to: 1.

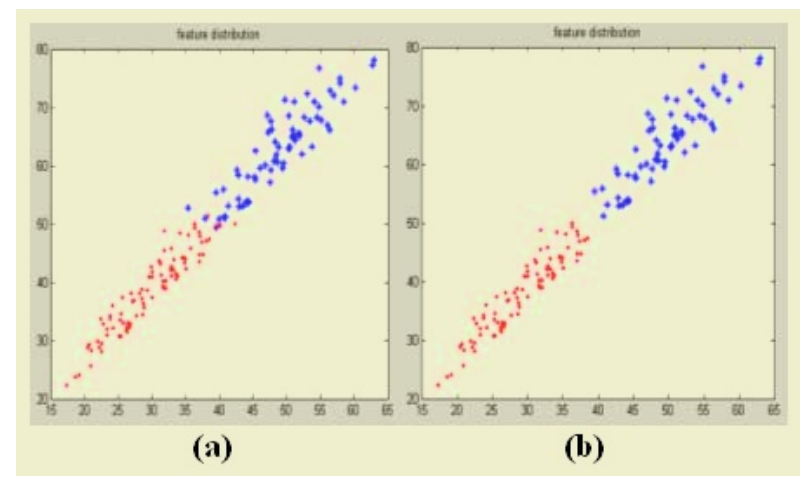

Fig. 1. The illustration of sample selection: (a) before and (b) after sample selection. Where x-axis: $\bar{\varrho}$ of $\nu=0, \mu=1$ and y-axis: $\bar{\varrho}$ of $\mathrm{v}=3, \mathrm{u}=5$

Fig.1.(b) shows the result of Fig.1.(a) by the editing algorithm. From this figure we can see that the intersectional points are discarded. This decreases the affect of ambiguous samples for voting procedure. In the next section, we will show that after sample selection the categorization accuracy has been improved to a certain extent.

\section{Experimental Results}

In our experiment, we use a database with 392 traditional Chinese painting images, which includes 132 flower-and-bird paintings, 127 figure paintings and 133 landscapes. These images are painted by different painters in different dynasty. Some works by modern painters are also included. The image size varied from $200 \times 200$ to $500 \times 500$. Some samples are shown in Fig. 2 .

We use the leave-one-out rule of cross validation to test our approach. Every time we select one as the test image and the others are as reference samples. This process repeats throughout the whole experimental data. During relativedistance based voting, we need to determine the threshold for voting. The threshold will decide whether the sample casts a vote of class $m$ to the test image. We compute average dispersivity of the selected training samples as the threshold, i.e.

$$
\text { Threshold }=d / v
$$




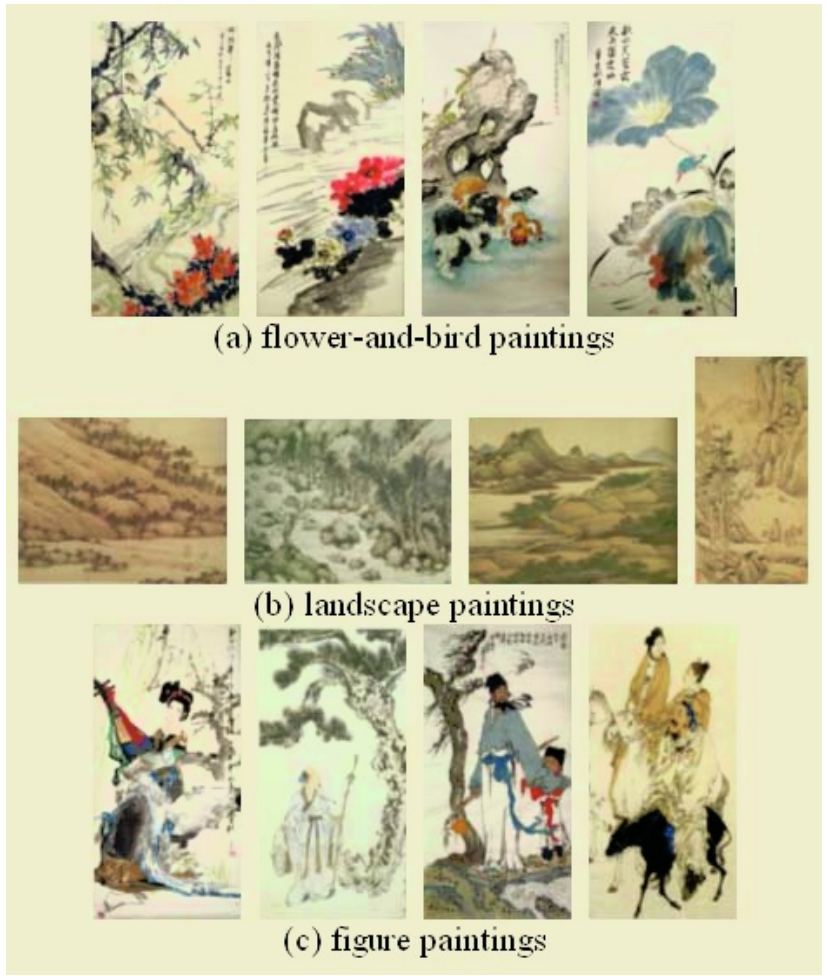

Fig. 2. Some samples of the experimental data

Where $d$ and $v$ are for the average mutual distance and variance of samples feature vectors.

The comparison with different features is carried out. The categorization results are shown in Table.1. presented in the confusion matrix form. In the class confusion matrix $A=\left(a_{i j}\right), a_{i j}$ means the samples number of class $i$ that predicted into class $j$. The diagonal elements are the correct classification numbers. The non-diagonal elements are the numbers of false categorization. Table.1. demonstrates that the scheme combining the statistical Gabor feature and color feature $(G C)$ has improved the categorization accuracy comparing with the single feature $(G$ or $C)$. The sample selection $(G C+)$ farther enhances the classification result.

\section{Conclusion}

In this paper, we present an automatic classification scheme. The statistical Gabor feature and color histogram are combined to classify the traditional Chinese painting images. And we use relative-distance based voting rule to finally categorize the images. The Gabor feature, description of local texture information of image, is compensated with the absence of spatial information, the drawback 
Table 1. The confusion matrix of landscape, figure and flower-and-bird paintings using various features. C: color histogram; G: Gabor feature; GC: combining Gabor feature and color histogram without sample selection; $\mathrm{GC}+$ : combining Gabor and color histogram after sample selection

\begin{tabular}{|l|l|c|c|c|}
\hline & Feature Used & Landscape & Figure & Flower-and-bird \\
\hline \multirow{5}{*}{ Landscape } & C & 110 & 23 & 0 \\
\cline { 2 - 5 } & G & 111 & 21 & 1 \\
\cline { 2 - 5 } & GC & 119 & 14 & 0 \\
\cline { 2 - 5 } & GC+ & 124 & 9 & 0 \\
\hline \multirow{5}{*}{ Figure } & G & 1 & 92 & 34 \\
\cline { 2 - 5 } & G & 4 & 92 & 31 \\
\cline { 2 - 5 } & GC & 1 & 100 & 26 \\
\cline { 2 - 5 } & GC+ & 0 & 106 & 21 \\
\hline \multirow{5}{*}{ Flower-and-bird } & G & 1 & 36 & 95 \\
\cline { 2 - 5 } & G & 3 & 35 & 94 \\
\cline { 2 - 5 } & GC & 1 & 19 & 112 \\
\cline { 2 - 5 } & GC+ & & & \\
\hline
\end{tabular}

of global color histogram. The preliminary experimental results have shown the superiority of our method.

\section{References}

1. M. Swain, D.Ballard. Color indexing. International Journal of Computer Vision, 7(1): 11-32, 1991.

2. G.Pass, R.Zabih, J.Miller. Comparing images using color coherence vectors. ACM Multimedia. Boston, MA, 1996.

3. M.Striker, M.Orengo. Similarity of color images, SPIE proceedings, 1995.

4. Y.L.Ho, K.L.Ho, H.H.Yeong. Spatial color descriptor for image retrieval and video segmentation. IEEE Transaction on multimedia. 5(3), 2003.

5. J.Z.Wang, J.Li, G.Wiederhold. SIMPLIcity: Semantics-sensitive integrated matching for picture libraries. IEEE PAMI, 23(9):947-963, 2001.

6. W.Y.Ma, B.S.Manjunath. Texture features and learning similarity. IEEE CVPR, 1996.

7. H.Yu, W.Wolf. Scenic classification methods for image and video databases. SPIE Proceeding, vol.2606, 363-371, October 1995.

8. S.Belongie, C.Carson, H.Greenspan, J.Malik. Color and texture-based image segmentation using EM and its application to content-based image retrieval. IEEE ICCV. pp.675-682, Jan. 1998.

9. J.Huang, S.Ravikumar, R.Xabih. An automatic hierarchical image classification scheme. Proc. ACM multimedia, Sep. 1998.

10. S.Jiang, W.Gao, W.Wang. Classifying traditional Chinese painting images. IEEE PCM, pp.1816-1820, Dec. 2003.

11. C.Liu, H.Wechsler. Gabor feature based classification using the enhanced fisher linear discriminant model for face recognition. IEEE Trans. IP, 11(4):467-476, 2002.

12. G.M.Haley, B.S.Manjunath. Rotation-invariant texture classification using modified Gabor filters. Proceedings of the International Conference on Image Processing. Vol.1, 1995. 
13. L.Chen, G.Lu, D.Zhang. Effects of different gabor filter parameters on image retrieval by texture. Proc. 10th Int'l Conf. Multimedia Modeling, 2004.

14. R.O.Duda, P.E.Hart, D.G.Stork, Pattern classification, Wiley, October 2000.

15. W. Liu ed. Traditional Chinese paintings thesaurus, pp.392-399, Huawen publisher, 1990 (in Chinese). 